\title{
A Review of a Mechanical Vapour Compression Refrigeration System: Environment and Performance Challenge
}

\author{
Yaser S. Mahmood ${ }^{1 *}$, Nooraldeen Salih ${ }^{1}$, Raid A. Mahmood ${ }^{1,2}$, Ahmed M. Daabo $^{3}$ \\ yaseralmola@uomosul.edu.iq \\ ${ }^{1}$ Mechanical Engineering Department, College of Engineering, University of Mosul, Iraq. \\ ${ }^{2}$ College of Enginering, University of Zakho, Kurdistan Region, Iraq. \\ ${ }^{3}$ Mining Enginering Department, College of Petroleum and Mining Engineering, University \\ of Mosul, Iraq.
}

\begin{abstract}
Environmental issues and energy cost have become important global concerns, receiving much attention and prompting consideration of alternative energy sources. This paper presents a comprehensive review of various theoretical and experimental studies which were carried out in air conditioning, refrigeration, and heating application. It explores a deeper understanding of mechanical vapour compression systems and alternative refrigerants that could be used instead of HCFC and CFC in future systems. Also, this paper considers studies that reported using new alternative refrigerants in refrigeration and air conditioning systems in both domestic and industrial sectors. This paper concludes that alternatives such as mixtures and natural refrigerants play a crucial role in reducing the environmental impacts of CFC and HCFC refrigerants. These alternatives show effective performance when used in refrigerant and air conditioning systems. Finally, this paper presents a future vision of the required systemic change.
\end{abstract}

Keywords: Alternative refrigerants, Mixture refrigerants, vapour compression refrigeration system, vapour injection technique, gravity flash tank, Ozone depletion potential (ODP).

\section{Introduction}

Air-conditioning, heat pump and refrigeration systems are widely used. These systems have similar components although they have different applications (Rasmussen 2011). Refrigerants are used as a working fluid in these systems to provide the refrigeration or heating requirements by transfer of heat between low and high-temperature sinks. However, there are many different varieties of refrigerants which are used as the working fluid in these systems. The refrigerant selection depends on the application required while taking the environmental effect into account. The majority of the refrigerants have excellent thermodynamic properties which are useful in mechanical vapour compression systems (Mohanraj et al. 2009). The mechanical vapour compression system (MVCS) is one of the refrigeration and/or air conditioning systems that can be used in domestic and commercial applications, but because of increased environmental concerns, refrigerant selection for the mechanical vapour compression system face challenges. Global warming concerns are growing in Australia and other developed countries. This is a serious concern for refrigerants used as the working fluid in mechanical vapour refrigeration systems (Bryson et al. 2011), as they have a substantial greenhouse gas effect if released into the atmosphere. Therefore, to reduce the impact of the refrigerant on the environment while maintaining a good performance, refrigerant selection and the system design need be considered in conjunction. Siva Reddy et al. (2012) presented a theoretical evaluation 
of system performance by using different refrigerant such as "R134a, R143a, R404A, R407C, R410A, R502, and R507A" to obtain optimum system performance. From an energy point of view, environmental concerns can be ameliorated if renewable sources are employed. In order to make the heat pump more effective researchers are working to overcome the rapidly increasing cost of energy, and reduce the emission of polluting substances. Heat pumps have applications for heating, cooling and humidity control in air conditioning systems. According to Nguyen et al. (2001), "Vapour compression heat pumps are refrigeration systems whose operational cycle requires work input to produce transfer of heat from a lower temperature source to higher temperature sink". Vapour compression systems contribute to a reduction of environmental impact by producing a comparative reduction in energy use compared with other energy sources such as boilers fired by fossil fuels or electrical radiator. Some researchers use an experimental approach while others depend on theoretical work to evaluate their ideas. This paper provides a review of various theoretical and experimental studies which were carried out in air conditioning, refrigeration, and heating applications, and opportunities for development of mechanical vapour compression systems are identified.

\section{Refrigerants Types:}

There are many different types of refrigerants which are used in mechanical vapour compression refrigeration systems. Some of these refrigerants are pure substance, whereas others are mixtures. Generally, the refrigerants can be classified into Hydrochlorofluorocarbons (HCFC), Hydrofluorocarbons (HFC), Chlorofluorocarbons (CFC), and Hydrocarbons HCs (Leelananda 2007). However, some of these refrigerants have been phased out as a result of the Montreal Protocol, signed in 1987, due to their ozone depletion effect. As a result, in many developed countries, some refrigerants have been prohibited for use in new mechanical vapour compression systems from 2010 (Aprea et al. 2011). Also, according to the Kyoto Protocol of 1997, which proposed measures to alleviate global warming issues, (CFC) and (HCFC) refrigerants have a significant effect on the environment. Therefore, in developed countries such as Australia, Chlorofluorocarbons (CFC) are not used as a working fluid in mechanical vapour compression refrigeration systems because of high ozone depletion effect (Cabello et al. 2004). Furthermore, Hydrochlorofluorocarbons (HCFC) are being replaced. They have been used in commercial and domestic systems with different application temperatures. Consequently, Hydrofluorocarbons HFCs refrigerant are the best choice to use in mechanical vapour compression refrigeration system as a replacement for (CFC) and (HCFC) refrigerants (Fernández-Seara et al. 2010). La Rocca and Panno (2011) reported that as (HFC) refrigerants do not have a damaging effect on the ozone layer and do not contain chlorine, (HCFC) are being replaced with (HFC) refrigerants. As a result, 2040 will see the end of (HCFC) refrigerants in developing countries. In developed countries, the use of (HCFC) refrigerants will cease before 2030. (Powell 2002) presents the detailed program of withdrawal of particular refrigerants from service.

\section{Mixtures of refrigerants:}

As per Montreal protocol of 1987 and regulation number 2037/200 of the European parliaments in 2000, the reduction in use of CFC and HCFC refrigerants have commenced (Leelananda 2007), (La Rocca \& Panno 2011). Consequently, the use of environmentally friendly mixture refrigerants that provide good thermodynamic and physical properties became the suitable solution to reduce adverse environmental effects. Mixed refrigerants are a mixture of HCs, HCFCs, and HFCs refrigerants in specific weight ratio, which can be used safely in mechanical 
vapour compression systems at different application temperatures. For example, to replace the refrigerants HCFC-22 (R22) and CFC-502 (R502), the refrigerant mixtures "R507A (R125/ R143a, 50\% / 50\% wt\%), R410A (R32/R125, 50\% / 50\%, wt\%), R407C (R32 / R125 / R134a, 23\% / 25\% / 52\%, wt \%), and R404A (R125 / R134a / R143a, 44\% / 4\% / 52\%, wt\%)" are recommended (Geller et al. 2001). In addition, $t$ refrigerant mixtures are harmless for the ozone layer. The refrigerant mixtures can also be classified to Zeotropic mixture (non-azeotropic mixture), Azeotropic mixture, and Near azeotropic mixture (Didion \& Bivens 1990).

\subsection{Zeotropic mixture (Non-azeotropic mixture).}

A zeotropic mixture is not a pure refrigerant; it consists of two or more refrigerant components. The behaviour of a zeotropic mixture refrigerant is not like the behaviour of a single refrigerant during phase change due to glide temperature (Mohanraj et al. 2011). The glide temperature can be defined as the temperature difference between the bubble and dew point temperatures at a given pressure and composition. Therefore, the glide temperature influences the phase change process such as condensation and evaporation processes, (Leelananda 2007). In addition, the glide temperature provides a reduction of entropy generation in a condenser and an evaporator due to matching between the heat transfer and glide temperature of the zeotropic mixture refrigerant (Venkatarathnam et al. 1996). Mohanraj et al. (2010) reported that because the glide temperature depends on the pressure drop in the condenser and evaporator producing a glide temperature range from $4^{\circ} \mathrm{C}$ to $7^{\circ} \mathrm{C}$, the condensation and evaporation processes are not isothermal processes. Furthermore, Giuliani et al. (1999) stated that the behaviour of the zeotropic mixture refrigerant effects the vapour compression system performance in different parts of the system due to changes in circulation composition. Indeed, the zeotropic mixture refrigerants behave differently in the vapour compression system as a result of these composition changes.

\subsection{Azeotropic mixture:}

Azeotropic mixture is a refrigerant which consists of two or more pure refrigerants, and cannot be separated or dissolved during the phase change processes. So, the behaviour of azeotropic mixture refrigerants is more like that of a single refrigerant. In addition, the boiling point temperature of the azeotropic mixture refrigerants is lower than their constituents (Mohanraj et al. 2011). Further, azeotropic mixture refrigerants can be used for different application temperatures such as commercial refrigeration systems and domestic refrigeration systems. For this reason many researchers used the azeotropic refrigerant as an alternative refrigerant. Sami and Aucoin (2003) conducted an experimental work to assess the impact of liquid injection in an "air source heat pump system using an azeotropic mixture refrigerant". The results showed that liquid injection at various ratios has a positive effect on dropping the "discharge temperature" and the head pressure which offers compressor protection benefits. Llopis et al. (2010) presented an experimental work to compare the energy performance of both a nearazeotropic refrigerant and an azeotropic refrigerant in a double stage compression refrigeration plant with sub-cooler heat exchanger. The results showed that the azeotropic refrigerant is better than the near-azeotropic refrigerant for low evaporator temperature applications. In addition, the coefficient of performance of both refrigerants is similar when the sub-cooler system is used. This indicates the azeotropic mixture refrigerants have stability of composition during the phase change processes, which is a significant factor in mechanical vapour compression systems. 


\subsection{Near azeotropic mixture:}

The behaviour of near azeotropic mixture refrigerants is similar to the behaviour of azeotropic mixture refrigerants. So, the hardware of the vapour compression system does not need to be altered. However, the near azeotropic mixture refrigerants have very low glide temperature ranges which are from $0.2^{\circ} \mathrm{C}$ to $0.6^{\circ} \mathrm{C}$ (Mohanraj et al. 2011). Therefore, refrigerant composition may change during the phase change processes. Arora and Kaushik (2008) reported that the exergetic efficiency of the azeotropic mixture refrigerants is better than that of near azeotropic mixture refrigerants. In addition, the coefficient of performance for the azeotropic mixture refrigerant in a vapour compression system is better than that of near azeotropic mixture refrigerant, particularly once the "pressure drop in the condenser and evaporator is increased".

\section{Thermodynamic properties of mixture refrigerants:}

The thermodynamic properties are the most important factors that should be considered to provide the system performance requirements. The thermodynamic properties need to be chosen to produce reliability in the system. Because the applications of mechanical vapour compression systems differ, the refrigerants must be able to accommodate a range of application design temperatures. Therefore, to obtain low discharge temperatures and to operate a vapour compression system at low application temperatures, the specific heat and the boiling temperature of the refrigerant should be low. In addition, to achieve an operating condition of the condenser in a vapour compression system at a point removed from the critical point on the pressure and enthalpy diagram, the critical temperature of the refrigerant should be high. Furthermore, to obtain a functional refrigerant effect, the critical pressure should be low. Another thermodynamic property that should be considered is the molecular weight. Because of the directly proportional relationship between the molecular weight and specific volume, the molecular weight will directly govern the choice of the compressor size. To provide an alternative refrigerant mixture that gives a suitable system performance as that of the existing halogenated refrigerant, the thermodynamic properties of the chosen mixtures should include the system operating temperature.

\section{Thermo-physical properties of refrigerant mixtures:}

The thermo-physical properties should be considered to provide successful condensation and evaporation processes using refrigerant mixtures in mechanical vapour compression systems. Thermal conductivity and viscosity are the most significant properties to achieve effective condensation and evaporation processes. At high values of thermal conductivity of both liquid and vapour phases of the refrigerant mixture, the condenser and evaporator will operate effectively due to the high value of the heat transfer coefficient (Mohanraj et al. 2011). Consequently, the power consumption will be reduced. Geller et al. (2001) presented experimental work to measure the thermal conductivity of different refrigerant mixtures such as R404A, R407C, and R507A at temperature range from $250 \mathrm{~K}$ to $400 \mathrm{~K}$. Liquid density is another physical property that will effect on the refrigerant charge requirement. An applicable model which is suitable to calculate the thermal conductivity of superheated vapour, saturated liquid and vapour at point removed from the critical point is required. Therefore, viscosity and liquid density need be considered when choosing a refrigerant mixture as an alternative refrigerant the thermal conductivity. 


\section{Refrigerant environment properties:}

Ethane and methane are essential gases to generate the halogenated refrigerants which are a chemical family derived from hydrocarbons. The majority of the halogenated refrigerants have chlorine and fluorine atoms. However, these atoms have an impact on the environment which can be evaluated by "ozone depletion potential (ODP) and global warming potential (GWP)" (Kumar \& Rajagopal 2007).

\subsection{Ozone depletion potential (ODP) and Global worming potential (GWP):}

Two of the important factors that should be considered in selecting the refrigerant type are the "ozone depletion potential (ODP) and global warming potential (GWP)". The ODP of a refrigerant represents the refrigerant's capacity to destroy the ozone layer. Molina and Rowland (1974) asserted that chlorine is an essential substance in refrigerants, but it is able to reach the stratosphere and destroy the ozone layer where it acts as a catalyst. In contrast, the GWP measures the energy balance between incoming energy from the sun that heats earth and the amount of energy that radiated from earth to space (Llopis et al. 2011). Mohanraj et al. (2009) asserted that more than $30 \%$ of the solar flux are reflected into space and the rest of the solar radiations are passed through the atmosphere to reach the ground and increase earth temperature. The ODP and GWP for some refrigerant mixtures and common refrigerants, respectively are given in (Kumar \& Rajagopal 2007),(Bolaji \& Huan 2013). It can be concluded from (Bolaji \& Huan 2013) the ODP and GWP do not have zero values for the same refrigerant: just the R718 (water/steam) and ammonia (R717) have that. However, La Rocca and Panno (2011) and Mohanraj et al. (2009) reported that refrigerants that have zero ODP with zero or low values of GWP will not harm the environment. Therefore, alternative refrigerant should have zero ODP and lower GWP to reduce the impact on the environment. In addition to water and ammonia are the ultimately environmentally friendly refrigerants.

\section{Mechanical vapour Compression Refrigerant system:}

A mechanical vapour compression refrigeration system can be used to provide both heating and cooling applications. The majority of domestic and commercial applications use a mechanical vapour compression system. However, the systems have different component sizes which match the system capacity that is essential to come across the demand of the application. Mechanical "vapour compression refrigeration system" can be classified as single stage and multi stage systems.

7.1 Single stage mechanical vapour compression system: A single-stage mechanical vapour compression system involves "four main parts the compressor, condenser, expansion valve and evaporator". These four main parts are connected systematically to complete a closed cycle as shown in (Rasmussen 2011). This cycle has four thermodynamic processes which are "isentropic compression, isobaric heat rejection (condensation), isenthalpic expansion, and isobaric heat absorption (evaporation)" as pointed in P-h diagram (Rasmussen 2011). The function of the mechanical vapour compression system is to "transfer heat from a low temperature zone to a high temperature zone". Indeed, the mechanical vapour compression system is a simple system that includes four essential parts to transfer the energy and meet application requirements at system design conditions. 
7.2 Multi stage mechanical vapour compression system: A multi-stage or two-stage vapour compression refrigeration system consists of more than one compressor to complete the compression process through "a multi-stage compression" process. In the multi stage system, there is an intercooler or economizer between the compressors to connect the "discharge line of the low stage compressor with the suction line of the high stage compressor" as illustrated in (Bertsch \& Groll 2008). In the multistage vapour compression refrigeration system, a flash tank is utilized to "subcool the liquid refrigerant to the saturated temperature corresponding to the interstage pressure" (Wang et al. 2000). The interstage pressure can be defined as the pressure located between high pressure of low stage compression and low pressure of high stage compression (Wang et al. 2000). Further, in the multistage compression system, the compression ratio of each stage is lower than that in the single-stage; however, the compression efficiency in the multistage compression is higher than that in the single-stage system. Therefore, it is recommended to use with high refrigeration system capacity.

\section{Theoretical and experimental studies:}

Many different theoretical and experimental studies have been completed. Some of these studies have considered the refrigerants and their alternatives to solve the environmental issues, while others have considered system components and system modifications to obtain the optimum coefficient of performance at a low energy cost.

8.1 Alternative refrigerants studies: The chlorofluorocarbons (CFC), and hydrochlorofluorocarbons (HCFC) refrigerants are being phased out because they have "high global warming potential (GWP) and Ozone depletion potential (ODP)" (Kumar \& Rajagopal 2007). Hydrofluorocarbons (HFC) refrigerants are being used instead of using the CFC and HCFC refrigerants. Many studies have been carried out to propose appropriate refrigerants as a "working fluid in mechanical vapour compression systems".

8.1.1 Use R507A mixture as an alternative refrigerant: R507A is a HFC, and it is an azeotropic refrigerant. It is also a blended refrigerant, consisting of $50 \%$ pentafluoroethane (HFC-125) R125 and 50\% trifluoroethane (HFC-143a) R143a (Llopis et al. 2010). R507A is one of the refrigerants used in vapour compression refrigeration system due to its good thermophysical and thermodynamics properties (Mohanraj et al. 2011). In addition, the R507A has zero ODP and low GWP which are essential factors for an environmentally friendly refrigerant. La Rocca and Panno (2011) reported that refrigerants that have zero ODP with zero or low values of GWP will not harm the environment. The R507A is classified to in a safe group (A1) (Arora \& Kaushik 2008). Furthermore, the R507A condenses and evaporates as a single substance, and does not have the glide temperature problem. Geller et al. (2001) presented experimental work to study measure the "thermal conductivity of refrigerant mixtures (R404A, R407C, R410A, and R507A)" at a temperature range from 250 to $400 \mathrm{~K}$. The results showed that the mixtures are appropriate for superheated vapour, saturated vapour and vapour at a point removed from the critical point. Result also showed that these refrigerant mixtures are suitable to replace $\mathrm{R} 22$ and $\mathrm{R} 502$.

Llopis et al. (2010) presented experimental work to compare the energy performance of both R404A and R507A refrigerants in a double-stage vapour compression refrigeration system with sub-cooler heat exchanger. The results showed that R404A has good performance at a high evaporator temperature application and R507A is suitable to use in low-temperature applications. Bolaji (2011) investigated the performance of R404A and R507 in window type air conditioner system experimentally. The result showed that the average coefficient of performance of R507 is increased by $10.6 \%$ when compared with that of R22. However, the 
coefficient of performance for R404A is reduced by $16.6 \%$ when compared with that of R22. Parekh and Tailor (2012) presented a theoretical work to analyse the "performance of a cascade system" by using an ozone friendly refrigerant pair. The results reveal that COP of the system increased from 0.7851 to 1.232 when the evaporator temperature is increased from $-80^{\circ} \mathrm{C}$ to$50^{\circ} \mathrm{C}$ with the other parameters kept constant. However, the COP is decreased from 0.9274 to 0.5486 when the condenser temperature is increased from $25^{\circ} \mathrm{C}$ to $50^{\circ} \mathrm{C}$.

8.1.2 Other alternative refrigerants: Different studies have presented alternative refrigerants' behaviour in mechanical vapour compression refrigeration systems in order to provide environmentally friendly refrigeration covering many different applications. These studies covered single and two stage systems cooling and/or heating applications.

Aprea and Greco (2003) evaluated compression performance between hydrochlorofluorocarbons HCFC (R-22) and hydroflourocarbons HFC (R407) by using a reciprocating compressor in a vapour compression refrigeration system experimentally. The experiment involved a sime-hermatic compressor, "water-cooled condenser, liquid receiver, thermostatic expansion valve, and plate finned tube evaporator". The evaporator temperature range and the condenser temperature of the experiment were $\left(-30^{\circ} \mathrm{C}-\left(-5^{\circ} \mathrm{C}\right)\right)$ and $35^{\circ} \mathrm{C}$, respectively. The results of the experiment revealed that $\mathrm{R} 22$ performance is better than $\mathrm{R} 407 \mathrm{C}$ because the isentropic and volumetric efficiency of the semi-hermetic compressor with R22 are better than that of R407C. Kumar and Rajagopal (2007) conducted experimental and computational analysis work to develop an eco-friendly refrigerant mixture, which involved hydrochloroflurocarbons R123 and hydroflurocarbons R290, used instead of HCFSs. In the experiment, the vapour compression refrigeration system used a hermetic reciprocating compressor with the refrigerant mixture which contained 70\% R129 and 30\% R290. The result of this study showed that the coefficient of performance of a refrigeration system with this refrigerant mixture is higher than that of R-12. In addition, the discharge temperature of the refrigerant mixture at the outlet of the compressor is lower than that of R12. Bertsch and Groll (2008) simulated and compared three models of an air source heat pump system by using R410A as a refrigerant at low ambient temperature. The simulation and comparison involved an intercooler, economizer, and cascade system models. The simulation results showed that worse efficiency values are obtained at the intercooler system mode. Jung (2008) presented the chlorofluorocarbons (CFC) and hydrochloroflurocarbons (HCFC) refrigerants characteristics and compared that with the Hydroflurocarbons (HFC) refrigerants theoretically. CFC and HCFC have excellent thermodynamic properties, chemical properties, and stability. However, they have negative effects on the environment such as ozone layer depletion and increasing global warming. Further, the Montreal Protocol in 1987 and Kyoto Protocol in 1997 recommended reducing the global warming and ozone-destroying by phasing out the CFC and HCFC. Therefore, the study suggested using $\mathrm{HFC}$ as working fluids in refrigeration and air conditioning systems instead of the CFC and HCFC to save the environment. Aprea and Maiorino (2008) presented experimental work to evaluate the energy performance of a mechanical cooling system by using an internal heat exchanger with carbon dioxide $\mathrm{CO}_{2}$ as a working fluid. The experimental system involved a plate finned tube heat exchanger, semi-hermetic compressor, and an electronic back pressure valve. The results showed that using an internal heat exchanger in the cooling system enhances the coefficient of performance by $10 \%$. In addition, the experimental work results suggested that the internal heat exchanger must be used with carbon dioxide in the cooling system to cool the control area. Mastrullo et al. (2009) conducted experimental work to measure the heat transfer coefficient for carbon dioxide refrigerant R744 and compared the values with that of the hydrofluorocarbons refrigerant R134a used in commercial refrigeration. The results showed that values of heat transfer coefficients of R744 
are always higher than that of R134a. Also, the peak of the heat transfer coefficient is at low vapour qualities. Fernández-Seara et al. (2010) measured a heat transfer coefficient of outer condensation on a cupronickel tube by using the hydrochloroflurocarbons R22 and its retrofit substitutes such as R417A, R422A, and R422D experimentally. It was concluded that the Heat transfer coefficient increases were reported with raises in the wall sub-cooling temperature and Heat transfer coefficient for vapours R417A, R422A, and R422D are reported to be lower by $65-76 \%, 24-31 \%$, and $60-67 \%$ relative to the heat transfer coefficient for R22 respectively. Llopis et al. (2011) focused on the experimental comparison between hydrochloroflurocarbons HCFC refrigerant such as R22 and hydroflurocarbons HFC refrigerants such as R422A, and R417B by using a double stage vapour compression system which has an evaporator temperature range from $-31^{\circ} \mathrm{C}$ to $-17^{\circ} \mathrm{C}$ and condenser temperature range from $38^{\circ} \mathrm{C}$ to $48^{\circ} \mathrm{C}$. The results showed that the cooling capacity of the refrigeration system of R422A and R417B is higher than that of R22. Moreover, the coefficient of performance of refrigeration system when R422A and R417B are used is smaller than that of R22. Mohanraj et al. (2011) presented theoretical work that involved an extensive study to review a recent refrigerants mixtures which are being used in refrigeration, air conditioning, and heat pump units. Azeotropic, Near azeotropic, and Zeotropic mixtures properties were showed different properties such as thermodynamic, thermo-phiscal, chemical, and environmental in different evaporator temperature applications such as domestic, commercial, and automobile systems. According to the extensive study of different experimental and theoretical investigation of refrigerants mixtures, hydroflurocarbon and hydrocarbon $\mathrm{HFC} / \mathrm{HC}$ refrigerant mixture such as $\mathrm{R} 430 \mathrm{~A}$ is a good substitute for replacing R12 and R134a in the domestic small units. Moreover, the best refrigerants mixture that provides a good performance in an automobile system instead of R12 and R134a are R430A and R152a. Further, in the low evaporator temperature application, R507 is the best substitute for replacing R502. La Rocca and Panno (2011) conducted experimental work to compare the performance of hydrochloroflurocarbons (HCFC) refrigerants such as R22, R123, and R124 with hydroflurocarbons (HFC) refrigerant such as R422A, R422D, and R417A in a vapour compression refrigeration system. The results showed that discharge temperature of the compressor by using R417A, R422A, and R422D is less than that of R22; therefore, this advantage subjects the compressor to less thermal stress and offers longer life time. Heller et al. (2012) used dynamic light scattering (DLS) to measure the thermophysical properties of ternary refrigerant mixtures R417A (50\% R134a, 46.6\%R125 and 3.4\%R600a by mass) and R417B (18.25\% R134a, 79\%R125 and 2.75\%R600a by mass) which can be suggested to use as an alternative refrigerant. The thermophysical properties which involve surface tension and saturated liquid kinematic viscosity were determined simultaneously. At $253.15 \mathrm{~K}$ up to the critical point of refrigerant, the thermophysical properties were measured with uncertainties $1 \%$ to $2 \%$ for surface tension and $1 \%$ to $3 \%$ for kinematic viscosity. The results of this study are validated by comparison with the literature which showed the mass weighted and mass/molar weighted prediction methods. In addition, the computer softwares Solkane 7.0 and REFPROP are used to compare the predicted and experimental data. Wang et al. (2012) conducted experimental study to operate "an inverter heat pump system with HFC125 refrigerant near the critical point of the refrigerant". The results showed that values of COP decrease when the hot water temperature increase at any compressor frequency value. However, the heating capacity had increased when the frequency is changed from 50 to $110 \mathrm{~Hz}$. In addition, "the maximum exergy loss is in the compressor; in contrast, the minimum exergy loss is in the evaporator. Moreover, the maximum valve of the exergy efficiency is obtained when the hot water temperature is around $65^{\circ} \mathrm{C}$ ". Siva Reddy et al. (2012) presented theoretical work that deals with an exergy analysis of a vapour refrigeration system. The results showed that best values of 
coefficient of performance and exergy efficiency are when the system is used the R134a refrigerant, whereas the poor values are at R407C refrigerant. Han et al. (2012) presented refrigerant "HFC161 in a small scale refrigeration system as an alternative refrigerant of HFC410A". The researcher calculated and analysed the coefficient of performance of HFC161, HFC410A, and HFC32 under nominated working conditions. The theoretical results showed that coefficient of performance of HFC161 is $10 \%$ higher than that of HFC32 and $17.8 \%$ higher than that of HFC410A. However, the experimental results showed that "the coefficient of performance of HFC161 is $15 \%$ higher than that of HFC410A and $25 \%$ higher than that of HFC 32". In addition, the discharge temperature of HFC161 is lower than that of HFC32 and HFC410A.

\subsubsection{Natural refrigerant}

Water is an ultimate natural refrigerant (working fluid) that can be used in "refrigeration and air conditioning systems". Water is an "environmentally friendly refrigerant because it has a zero ozone depletion potential (ODP), zero global worming potential (GWP), non-toxicity, and nonflammability" (Wang \& Li 2007). Therefore, the water is classified as a safe refrigerant, falling into the A1 group as a refrigerant code (R718) (Kilicarslan \& Müller 2005), (Šarevski \& Šarevski 2012). The water also has excellent chemical and thermodynamic properties, so it can be used both below and above its freezing point (Kilicarslan \& Müller 2005). However, at the below freezing application, water needs to be coupled with protective solution such as propylene or ethylene glycol to prevent freezing during operation. R-718 (water/steam) has been used in many different applications of air conditioning and refrigeration and received attention as a natural refrigerant due to the recommendation of the Montreal Protocol to reduce environmental issues (Wang \& Li 2007). Early, the water had been used in adsorption, absorption, desiccant air conditioning systems as mentioned in Ellison et al. (1997), Pope and Fry (1997), and Ekambara et al. (2008) studies. However, industries face some difficulties to provide a powerful and efficient compressor. These difficulties include compressor's blades manufacture, so many different studies have been done to use water as natural refrigerant and to work with the industries to obtain efficient working of water in refrigeration and air conditioning systems $(\mathrm{Li}$ et al. 2011). Recently, R-718 (water/steam) is being used as natural refrigerant in mechanical compression refrigeration, turbocompresso, and ejector air conditioning and refrigeration systems as mentioned in studies by Li et al. (2011), Šarevski and Šarevski (2012), and Chen et al. (2013). These studies have revealed that among the refrigerants such as R717, R12, R22, R134a and R290, R-718 (water/steam) presents the high coefficient of performance (COP) at high evaporator temperature.

Kilicarslan and Müller (2005) presented a comparative study to compare "the coefficient of performance (COP) of one stage vapour compression refrigeration system" theoretically. The study used a "compression refrigeration system which includes a compressor, a condenser, expansion valve, and evaporator" to use different refrigerants for comparing such as R-718 (water/steam), R22, R134a, R12, and R290. The CoolPack 2001, software was also used in the study to simulate and obtain the results of the theoretical refrigeration system. The results showed that high value of the COP is obtained when the system used the water as a refrigerant as compared with other refrigerants.

Li et al. (2011) presented a theoretical study to use a counter rotating axial impellers to compress water as a refrigerant in a compression refrigeration system. A Multi-stage compressor with an intercooler strategy is used as to operate the system. CFD numerical approach was used to predict the thermodynamic capacity of the counter axial impeller. The results showed that coefficient of performance of the vapour compression system is increased around $30 \%$ by using water at full load compared to a conventional refrigerant such as R134a. The result also 
demonstrated that novel axial composite impeller has the ability to produce a high pressure ratio with workable isentropic efficiency to compress the water as a refrigerant.

Šarevski and Šarevski (2012) presented a theoretical study to estimate performance characteristics, fluid flow characteristic, and parameters of the centrifugal compressor by using water as a natural refrigerant. The results demonstrated that the possibility of using centrifugal compressor with the R-718 (water/steam) as natural refrigerant in refrigeration and air conditioning applications is very large, which can be presented from ten $\mathrm{kW}$ up to 1-2 MW.

Wang and Müller (2012) developed an integrated water vapour compressor by using a composite material wheel. The new design of the water vapour compressor reduced the number of parts and mass. So, by "integrating all rotating parts, a drive shaft" is no longer required. The author used "a Kevlar fiber with epoxy matrix as a composite material to design the impeller of the water compressor because this type of material has excellent toughness, ductility, impact resistance, light weight, and high strength". Therefore, by depending on the wheel size the cost of wheel creation is very low, possibly below ten dollars. A numerical simulation was used to simulate the water compressor by using the new design of the wheel (Impeller). The results showed that integrated compressor with the new design of impeller can be used in air conditioning and refrigeration systems by using the water as natural refrigerant with very high coefficient of performance.

\section{System modification by using injection technique:}

Mechanical vapour compression systems can be modified by using vapour injection technique. The vapour injection technique was presented in 1979 for a room air conditioning system (Xu et al. 2011a). In 1982 a flash tank was used in a refrigeration system as a liquid vapour separator by Ueno and Fukuhara (1982) to inject a vapour into a rotary compressor, and "an injection control valve was used to control the injection process in the refrigeration system". After that, refrigeration cycle with different forms of injection technique was used to meet application requirements. Therefore, refrigeration cycle with vapour injection was classified into vapour injected cycle by using flash tank and vapour injected cycle by using sub-cooler vapour (internal heat exchanger) (Heo et al. 2012) .

\subsection{System Modification by using Internal Heat Exchanger:}

Winandy and Lebrun (2002) used the R22 as a working fluid to analyse and model the gas liquid injection in vapour compression system by using a scroll compressor experimentally. Cho et al. (2003) evaluated experimental work to measure the "performance of an inverter scroll compressor with liquid refrigerant injection by using various compressor frequency, injection pressure, and injection location". The operating parameters and injection location were considered to present the "influence of the liquid refrigerant injection on the system performance". Guoyuan et al. (2003) proposed sub-cooling system by using a scroll compressor with supplementary inlet, which can effectively solve application requirements for sub-zero region weather. The experimental system was operated at $-15^{\circ} \mathrm{C}$ ambient temperature and $45^{\circ} \mathrm{C}$, $48^{\circ} \mathrm{C}$ condenser temperature. The result of this study showed that heating energy efficiency ratio (EER) is improved by a supplementary circuit; however, the power input of the air source heat pump is increased. Wang, B., Shi, W., et al. (2009) investigated the effects of refrigerant injection in a scroll compressor. The results showed that efficiency of scroll compressor reaches the tip at pressure ratio equals one. Wang, B.Shi, W., et al. (2009) developed refrigeration system with a gas injected scroll compressor model and proposed the component parameters and the effect of gas injection on the system numerically. The results also showed that compression work and compression ratio decrease when the injection position increases. Wang, X. et al. (2009) investigated experimental work of a two stage vapour compression system by 
using a vapour injected scroll compressor and R410A as a refrigerant (working fluid). The experiment also considered the overall system performance and the refrigerant flow control strategy point of view. The result of this study concluded that the cooling capacity gain is $15 \%$ with a $2 \%$ of coefficient of performance at the ambient temperature of $46.1^{\circ} \mathrm{C}$. Heo et al. (2012) conducted experimental work for a refrigerant injection heat pump. The apparatus of this study, involved twin rotary compressor, receiver tank, two electronic expansion valve EEV, this study investigated the effect of intermediate pressure and injection amount of refrigerant. The result concluded that optimum sub-cooler pressure ratio is 0.4 to 0.7 at heating capacity operation, and it is 0.7 to 0.8 at the coefficient of performance consideration. Further, the optimum injection ratio range was $0.11-0.15,0.16-0.22$, and $0.19-0.29$ at an outdoor temperature range $-15^{\circ} \mathrm{C}$, $5^{\circ} \mathrm{C}$, and $5^{\circ} \mathrm{C}$, respectively.

\subsection{System Modification by using Flash Tank:}

Jain et al. (2004) compared a three ton air conditioning and vapour "compression refrigeration system with and without vapour injection by using a scroll compressor theoretically". The result exhibited that coefficient of performance is increased by 6-8\% as compared with a basic system which was without vapour injection”. Elbel and Hrnjak (2004) conducted experimental work to advance a "performance of a refrigeration system by using Flash Gas Bypass (FGB)". The results of this study "concluded that FGB increased the coefficient of performance and the cooling capacity of the refrigeration system by $7 \%$ and 9\%, respectively". Ma and Zhao (2010) presented the characteristic of a heat pump system with a flash tank coupled with a scroll compressor. The results showed that heat pump is improved by flash tank, and the coefficient performance of the heat pump is 1.73 at the operation condition which included $-25^{\circ} \mathrm{C}$ evaporator temperature, $45^{\circ} \mathrm{C}$ condenser temperature, and $10 \mathrm{bar}$ intermediate pressure. Hwang et al. (2010) presented experimental investigation of vapour injection control strategy in a heat pump system. Jang et al. (2010) measured and analysed the "effects of phase separator injection and internal heat exchanger injection on the heating performance of a two stage heat pump system" by using an "inverter driven twin rotary compressor" experimentally. The results showed that coefficient of performance. Heo et al. (2010) studied the effect of flash tank vapour injection on the heating capacity and the performance of a two stage heat pump system at a low ambient temperature by using an inverter-driven twin rotary compressor. The study considered the compressor frequency range from $50 \mathrm{~Hz}$ to $100 \mathrm{~Hz}$ at low ambient temperature range $-15^{\circ} \mathrm{C}$, $-5^{\circ} \mathrm{C}$, and $5^{\circ} \mathrm{C}$. The experimental work of this study involved two twin rotary compressors, indoor and outdoor units, a flash tank with $240 \mathrm{~cm}^{3}$, and electronic expansion valve (EEV). The results of this study showed that the coefficient of performance "COP and heating capacity of the injection cycle are increased by $10 \%$ and $25 \%$ respectively at low ambient temperature". However, the power consumption in the injection cycle is higher than power consumption in the non-injection cycle. The study also showed that $80 \mathrm{~Hz}$ of frequency gives a compressor reliability to work. Heo et al. (2011) presented experimental work of vapour injection techniques for double expansion sub-cooler and flash tank to enhance the heating performance of an air source heat pump system. The results showed that the average heating capacities of the flash tank, flash tank sub-cooler, and double expansion sub-cooler cycles are higher by $14.4 \%, 6 \%$, and $3.8 \%$, respectively, compared with sub-cooler cycle. Mathison et al. (2011), developed the model of refrigerant injection port that is used to inject the refrigerant during the compression process in a scroll compressor instead of injecting the refrigerant between compression stages. The results showed that increasing the number of injection ports number in the scroll compressor reduces the number of the multi-stage compressor and its cost. Xu et al. (2011b) proposed novel control strategy of a heat pump system by using flash tank injection experimentally in both 
transient and steady state cases. The results showed that the superheated degree of the injected vapour can be used as the control signal in the upper expansion valve. Sirwan et al. (2011) modified absorption ejector cooling system by adding a flash tank between the condenser and the evaporator theoretically. The results showed that adding of the flash tank to the cycle improves the cooling effect and the capacity of the evaporator. XU et al. (2012) conducted experimental work to evaluate the performance of vapour injected heat pump with flash tank system by using R32 as a substitute of R410A. The result was found that coefficient of performance and capacity are improved to be between $1.2 \%$ to $5.7 \%$ and $1.8 \%$ to $7.4 \%$ respectively when the outdoor operation condition changed from $-18^{\circ} \mathrm{C}$ to $46^{\circ} \mathrm{C}$. Furthermore, the result revealed that vapour injection is helpful to reduce the discharge temperature.

\section{Numerical and visualisation Investigation studies}

Numerical simulation is another approach which can be used by researchers. The numerical investigation is used when the experimental investigation becomes expensive, complex and/or dangerous (Becker et al. 2014). So, most of the researcher prefer the numerical works to save a time and obtain accurate results. Parvareh et al. (2010) is one of the researchers who used numerical simulation to present the two-phase flow behaviour and improve the heat transfer in a horizontal tube. One of the effective numerical approaches is Computational Fluid Dynamic (CFD). In the CFD numerical simulation, many of differential equations are solved according to the boundary conditions and case setting (FLUENT 2012, Daabo et.al.). Therefore, many researchers used this methodology to improve the refrigeration system performance in terms of improve the two-phase flow behaviour, separation efficiency, heat transfer, two-phase flow pattern and refrigerant two-phase distribution in evaporator and header (Duan et al. 2014).

Duan et al. (2014) conducted a three-dimensional numerical study for non-isothermal stratified two-phase flow pattern in a circular inclined pipe. In the modelling, steady axial momentum and heat transfer equations with low Reynolds number were solved. In order to model the turbulent for eddy viscosity, K-epsilon model and grid refinement were used. Air-water mixture was used as a working fluid. Gas and liquid superficial velocity were used to cover velocity range from 1.5 to $4 \mathrm{~m} / \mathrm{s}$ and from 0.1 to $0.27 \mathrm{~m} / \mathrm{s}$ respectively. There results showed that there is good agreement between the numerical pressure gradient along the inclined pipe and some empirical correlation from literature.

\section{Future vision of the refrigeration and air conditioning systems}

The future vision of the "refrigeration and air conditioning systems" should serve both environment and application requirements and should provide a sustainable system which can be used without any negative effects on the environment and system's reliability which means effectiveness and efficiency of system. From the environmental point of view, the refrigerant (working fluid) type should be considered and selected according to the values of the ODP and GWP. According to Montreal Protocol control, in the 1 January 2016 the HCFCs refrigerants will be banned to use and in 1 January 2020 the HCFCs refrigerant will be phased out but allow up to $0.5 \%$ in service until 2030 of the existing refrigeration and air conditioning equipment in the developed countries, however, in 1 January 2040 the HCFCs refrigerant will be phased out completely in developing countries (Powell 2002). Therefore, the natural refrigerant types such as water, carbon dioxide, hydrocarbon, and ammonia will play a significant role in refrigeration and air conditioning equipment. Recently, Bolaji and Huan (2013) reported that natural refrigerants, which are environmentally friendly and the ultimate solution, are the most suitable working fluid in refrigeration and air conditioning systems. Sarkar et al. (2013) also presented 
theoretical and optimization study to operate the cascade refrigeration system by using natural refrigerants. The study revealed that there is suitable system performance when using natural refrigerants such as hydrocarbon refrigerants. Therefore, the natural refrigerants are being used more intensively in refrigeration and air conditioning studies. Meanwhile, from the system performance point of view, the system's reliability, and efficiency are most important to provide application requirements. Therefore, many studies have provided different techniques to enhance the system performance such as Wang, B., Shi, W., et al. (2009), Heo et al. (2011), Mathison et al. (2011) and Baek et al. (2014). The vapour injection is one of the effective techniques which is being used to obtain high performance of refrigeration system. However, it should be applied to natural refrigerants to cover the environmental and application needs. So, many studies are being started to provide a suitable refrigeration system by redesign the main component such as a compressor and/or add new part to be suitable to work with the natural refrigerant, such as Wang and Müller (2012) and Li et al. (2011). Indeed, the world have started to encourage the refrigeration and air conditioning studies (academic and industrial sectors) to obtain sustainable systems that have ability to provide the environmental and application requirements.

\section{Conclusion}

This work tries to review the theoretical and experimental studies that have considered the alternative refrigerants and the system performance to obtain the environmentally friendly refrigeration systems. Refrigerant types, thermodynamic, and thermo-physical properties are discussed in this paper. According to the previous studies and the Montreal Protocol of 1987 and the regulation number 2037/200 of the European Parliaments in 2000 the HCFC, CFC refrigerants will be forbidden to use in air conditioning and refrigeration systems due to their negative effects on the environment. Therefore, some alternative refrigerants are presented in this paper, with the literature revealing that the R123, R290, and R407C are suitable substitutes refrigerants to use instead of the R22 refrigerant and refrigerant mixture of 70\% R129-30\% R290 is substitute for replacing the R12 refrigerant. Further according to the literature, the mixture refrigerant such as R507A and natural refrigerants play "a crucial role for reducing the environmental impacts of CFC and HCFC refrigerant". Also they are efficient refrigerant to use in air conditioning and refrigeration equipment. System modification by using vapour injection technique is an effective technique to improve the system performance. It also provides low energy consumption and long life for the compressor due to reduce the thermal stress as a result of reducing the discharge temperature. Future vision of the refrigeration and air-conditioning system is discussed to focus on the future needs. Proposed future works are presented in this paper to provide study with natural refrigerant such as water/steam, air, carbon dioxide, and hydrocarbons. CFD numerical "modelling" could be useful approach to enable a good design of "flash tank" and other parts in refrigeration system such as evaporator and heaters. As a result, the information which is presented in this paper may be useful to the researchers who work in refrigeration and air conditioning sector.

\section{Acknowledgment}

The authors would like to the University of Mosul for supporting the present research study.

\section{References}

[1] Baek, C, Heo, J, Jung, J, Lee, E \& Kim, Y 2014, 'Effects of vapor injection techniques on the heating performance of a $\mathrm{CO}<\mathrm{sub}>2</ \mathrm{sub}>$ heat pump at low ambient temperatures', International Journal of Refrigeration, 
[2] Becker, A, Kapitz, M \& aus der Wiesche, S 2014, 'Numerical Simulation of Single Bubble Dynamics During Flow Boiling Conditions on a Horizontal Surface', Heat transfer engineering, vol. 35, no. 5, pp. 461-71,

[3] Bolaji, B \& Huan, Z 2013, 'Ozone depletion and global warming: Case for the use of natural refrigerant-a review', Renewable and Sustainable Energy Reviews, vol. 18, pp. 49-54,

[4] Duan, J, Gong, J, Yao, H, Deng, T \& Zhou, J 2014, 'Numerical modeling for stratified gas-liquid flow and heat transfer in pipeline', Applied Energy, vol. 115, pp. 83-94,

[5] Elbel, S \& Hrnjak, P 2004, 'Flash gas bypass for improving the performance of transcritical R744 systems that use microchannel evaporators', International Journal of Refrigeration, vol. 27, no. 7, pp. 72435 ,

[6] FLUENT, A 2012, '14.5, Theory Guide; ANSYS', Inc., Canonsburg, PA,

[7] Daabo, A. M., Al-Mola, Y. S., Al-Rawy, A. Y., \& Lattimore, T. (2019). State of the art single-objective optimization of small scale cylindrical cavity receiver. Sustainable Energy Technologies and Assessments, $35,278-290$

[8] Daabo, Ahmed Mahmood Abdullah. Stress analysis and aerodynamic optimization of small-scale compressed air turbines. Diss. University of Birmingham, 2018.

[9] Daabo, A. M., Hammo, K. E., Mohammed, O. A., Hassan, A. A., \& Lattimore, T. (2020). Performance investigation and design optimization of micro scale compressed air axial turbine for domestic solar powered Brayton cycle. Sustainable Energy Technologies and Assessments, 37, 100583.

[10] Guoyuan, M, Qinhu, C \& Yi, J 2003, 'Experimental investigation of air-source heat pump for cold regions', International Journal of Refrigeration, vol. 26, no. 1, pp. 12-8,

[11] Heo, J, Jeong, MW \& Kim, Y 2010, 'Effects of flash tank vapor injection on the heating performance of an inverter-driven heat pump for cold regions', International Journal of Refrigeration, vol. 33, no. 4, pp. 848-55,

[12] Heo, J, Kang, H \& Kim, Y 2012, 'Optimum cycle control of a two-stage injection heat pump with a double expansion sub-cooler', International Journal of Refrigeration, vol. 35, no. 1, pp. 58-67,

[13] Heo, J, Jeong, MW, Baek, C \& Kim, Y 2011, 'Comparison of the heating performance of air-source heat pumps using various types of refrigerant injection', International Journal of Refrigeration, vol. 34, no. 2 , pp. $444-53$,

[14] Hwang, Y, Xu, X, Radermacher, R \& Pham, H 2010, Control strategy of vapor injection cycle, Purdue University School of Mechanical Engineering, <http://docs.lib.purdue.edu/iracc/1066.>.

[15] Jain, S, Jain, G \& Bullard, CW 2004, Vapor Injection in Scroll Compressors, School of Mechanical Engineering, Purdue University Purdue 12-15 July, <http://docs.lib.purdue.edu/icec/1642〉.

[16] Jang, Y, Lee, E, Chin, S \& Ha, S 2010, Effect of flash and vapor injection on the air-to-air heat pump system, Purdue University School of Mechanical Engineering, 〈http://docs.lib.purdue.edu/iracc/1138>.

[17] Li, Q, Piechna, J \& Müller, N 2011, 'Thermodynamic potential of using a counter rotating novel axial impeller to compress water vapor as refrigerant', International Journal of Refrigeration, vol. 34, no. 5, pp. 1286-95,

[18] Ma, G \& Zhao, H 2010, 'Performance prediction for heat pump system with flash tank coupled with scroll compressor', International journal of ambient energy, vol. 31, no. 3, pp. 153-60, viewed 2012/01/25, [19] Mathison, MM, Braun, JE \& Groll, EA 2011, 'Performance limit for economized cycles with continuous refrigerant injection', International Journal of Refrigeration, vol. 34, no. 1, pp. 234-42,

[20] Parvareh, A, Rahimi, M, Alizadehdakhel, A \& Alsairafi, A 2010, 'CFD and ERT investigations on two-phase flow regimes in vertical and horizontal tubes', International Communications in Heat and Mass Transfer, vol. 37, no. 3, pp. 304-11,

[21] Powell, RL 2002, 'CFC phase-out: have we met the challenge?', Journal of fluorine chemistry, vol. 114 , no. 2, pp. 237-50,

[22] Sarkar, J, Bhattacharyya, S \& Lal, A 2013, 'Performance comparison of natural refrigerants based cascade systems for ultra-low-temperature applications', International Journal of Sustainable Energy, no. ahead-of-print, pp. 1-15,

[23] Sirwan, R, Ali, Y, Zaharim, A \& Sopian, K 2011, Effect of adding flash tank on the evaporator's thermal load of the combined ejector-absorption cooling system, World Scientific and Engineering Academy and Society (WSEAS), Penang, Malaysia. 
[24] Ueno, F \& Fukuhara, K 1982, Refrigeration system, 246,857, Google Patents, US, 17 Aug. 1982.

[25] Wang, B, Shi, W \& Li, X 2009, 'Numerical analysis on the effects of refrigerant injection on the scroll compressor', Applied Thermal Engineering, vol. 29, no. 1, pp. 37-46,

[26] Wang, B, Shi, W, Han, L \& Li, X 2009, 'Optimization of refrigeration system with gas-injected scroll compressor', International Journal of Refrigeration, vol. 32, no. 7, pp. 1544-54,

[27] Wang, J \& Müller, N 2012, 'Preliminary Design and Investigation of Integrated Compressor with Composite Material Wheel', Applied Composite Materials, vol. 19, no. 3-4, pp. 739-46,

[28] Wang, X, Hwang, Y \& Radermacher, R 2009, 'Two-stage heat pump system with vapor-injected scroll compressor using R410A as a refrigerant', International Journal of Refrigeration, vol. 32, no. 6, pp. 144251 ,

[29] Winandy, EL \& Lebrun, J 2002, 'Scroll compressors using gas and liquid injection: experimental analysis and modelling', International Journal of Refrigeration, vol. 25, no. 8, pp. 1143-56,

[30] Xu, X, Hwang, Y \& Radermacher, R 2011a, 'Refrigerant injection for heat pumping/air conditioning systems: Literature review and challenges discussions', International Journal of Refrigeration, vol. 34, no. 2, pp. 402-15,

[31] 2011b, 'Transient and steady-state experimental investigation of flash tank vapor injection heat pump cycle control strategy', International Journal of Refrigeration, vol. 34, no. 8, pp. 1922-33,

[32] XU, X, HWANG, Y, RADERMACHER, R \& Pham, HM 2012, Performance Measurement of R32 in Vapor Injection Heat Pump System, Purdue. 